\title{
Distribution of non-plasma protein components in meconium from healthy and cystic fibrosis neonates
}

\author{
HC RYLEY \\ From the Department of Medical Microbiology, Welsh National School of Medicine, Heath Park, \\ Cardiff CF4 $4 X N$, UK
}

SUMMARY The distribution of four antigens of alimentary tract origin was studied in meconium collected from healthy and cystic fibrosis (CF) neonates. Two antigens, carcinoembryonic antigen and a $\beta$ glycoprotein designated Mec 6 , had significantly lower concentrations in the meconium from CF neonates compared with that from healthy full-term infants. As their concentrations were unrelated to whether or not the infants had had pancreatic insufficiency at birth, their measurement may be of value for screening. Their concentrations also depended on the gestational age of the newborn, and it is speculated that the CF lesion may affect the maturation of intestinal secretions.

The analysis of meconium for elevated albumin content is the most common routine screening procedure for cystic fibrosis (CF) in the newborn, but it is now clear that such screening procedures have a relatively high false negative incidence. ${ }^{1-3}$ Albumin levels in meconium reflect the pancreatic status of the newborn, and in a recent study in this laboratory about $25 \%$ of neonates with CF were found to have 'normal' pancreatic function at birth. ${ }^{3}$ Consequently, a more satisfactory test is needed to reduce this false negative incidence.

The nature of the CF lesion is still unknown, but one of its major effects is the production of 'abnormal' mucus, which directly mediates the major alimentary and pulmonary complications associated with the disease. Whether the abnormal nature of the mucus is due to changes in organic or inorganic composition, or solely to a lower than normal water content, is not known; there is some evidence for each of these aspects. ${ }^{4}$ Tests on meconium for CF screening depend on the detection of meconium compositional differences in CF compared to healthy meconium but, apart from the changes in serum protein content, few other aspects of meconium composition have been studied. 5 Antonowicz et al. ${ }^{6} 7$ found a significant change in the concentration of some glycosidase enzymes in CF meconium compared to normal meconium although only 'true positive' and 'true negative' specimens were compared. It is the purpose of this report to describe the distribution of some water-soluble meconium antigens of non-plasma origin.

Accepted for publication 30 July 1980
Seven such antigens were detected in normal meconium by bidimensional immunoelectrophoresis and, until further characterised, were named Mec 1 to Mec $7^{8}$ (Fig. 1a). Mec 1 and Mec 6 were an $\alpha$ and a $\beta$ glycoprotein, respectively, Mec 2 and Mec 7 were related to the mucin blood group substances, Mec 3 was identified as carcinoembryonic antigen (CEA), and Mec 4 as alkaline phosphatase. Preliminary studies indicated that there was a large decrease in their concentration in meconium from CF infants who had had pancreatic insufficiency at birth (Fig. 1b). Thus the distribution of four of the antigens, Mec 1, Mec 6, CEA, and alkaline phosphatase, was investigated in normal and CF meconium by immunoelectroassay. As a low level of the antigens in CF meconium might be due to the absence of intrinsic proteolytic release of antigens bound to the insoluble mucin gel phase, the distribution of the antigens was estimated before and after proteolysis of the meconium specimen with trypsin. Changes in the antigen composition in meconium from the fetus and preterm infants were also studied to determine if the time of gestation affected their final distribution, and the results obtained were related to their value in $\mathrm{CF}$ screening tests.

\section{Methods and material}

SPECIMENS

Meconium specimens were obtained during routine screening or had been sent from other hospitals in the UK. They were classified into four groups.

Group 1 (true negative) was meconium from 


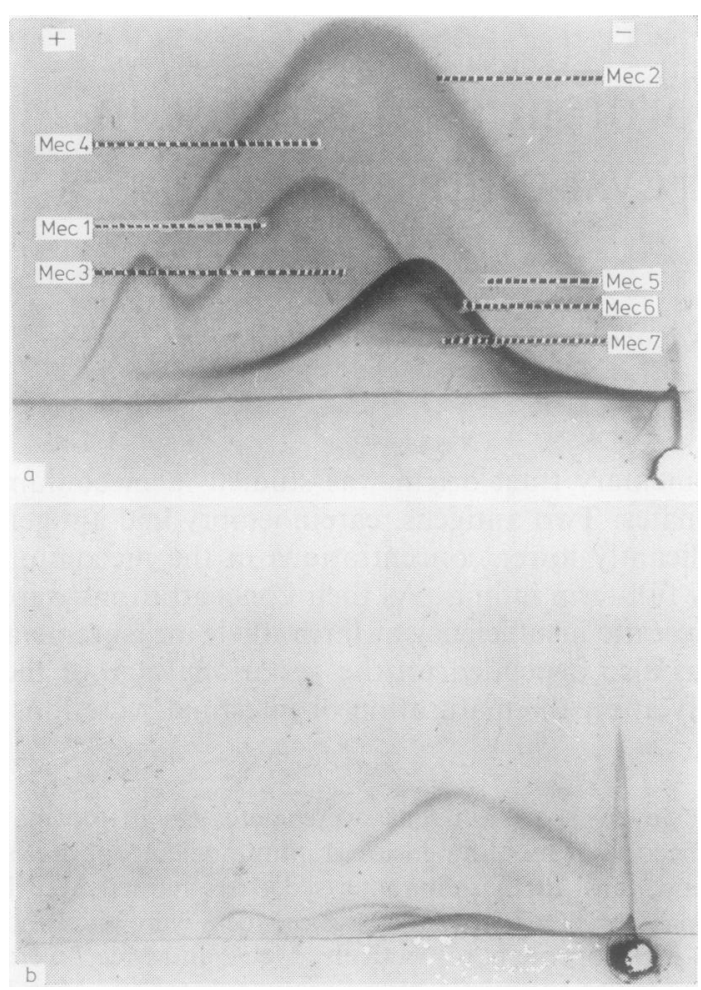

Fig. 1 Bidimensional immunoelectrophoresis of (a) $1 \%$ aqueous extract of meconium from a healthy infant; and (b) $1 \%$ aqueous extract of meconium from a CF infant against antiserum to meconium antigens of non-plasma origin.

healthy full-term infants with an albumin content in meconium specimen of less than $0.1 \mathrm{mg}$ per $\mathrm{g}$ dry weight. Group 2 (false positive) was meconium from healthy infants but with an albumin content of more than $50 \mathrm{mg} / \mathrm{g}$ dry wt. Group 3 (true positive) was meconium from infants with $\mathrm{CF}$ and an albumin content in excess of $50 \mathrm{mg} / \mathrm{g}$ dry wt. Group 4 (false negative) was meconium from infants with $\mathrm{CF}$ but with an albumin content of less than $20 \mathrm{mg} / \mathrm{g}$ dry wt. Diagnosis of CF was made on the basis of clinical symptoms together with a positive sweat test. ${ }^{9}$

Meconium specimens were also collected and grouped on the basis of estimated gestational age. Specimens from fetuses were grouped according to estimated gestational ages of 12 to 16 weeks and 17 to 20 weeks. The fetuses had been aborted therapeutically for various reasons, and estimated gestational age was based on crown-to-rump measurement. Three meconium specimens were obtained from fetuses of estimated gestational age 21 to 26 weeks after accidental miscarriages. Further specimens from premature infants were divided into two further groups on the basis of estimated gestational ages of 27 to 31 weeks and 32 to 36 weeks, respectively. A final group of meconium specimens was obtained from infants considered to have been born at full term; estimated gestational ages were greater than 38 weeks. The gestational age of the newborn infants was estimated by the obstetricians and paediatricians in the wards and was considered to be accurate to \pm 2 weeks.

PREPARATION OF SPECIMENS FOR ANALYSIS All specimens were frozen within 24 hours of being received and lyophilised. The dried material was stored at $-20^{\circ} \mathrm{C}$ before analysis. ${ }^{9}$ A $1 \%$ suspension of each specimen was made up in a $50 \mathrm{~mm}$ Tris, $31.7 \mathrm{~mm} \mathrm{HCl}$ buffer, $\mathrm{pH} 8 \cdot 1$, containing $150 \mathrm{mmol} / \mathrm{l}$ $\mathrm{NaCl}, 1 \mathrm{mmol} / \mathrm{l} \mathrm{CaCl}$, and $1 \mathrm{mg}$ per $\mathrm{ml}$ of bovine trypsin (Type III, Sigma Chemical Co, Poole, Dorset) and incubated overnight at $37^{\circ} \mathrm{C}$. A second suspension of each specimen was prepared in the same buffer, but without trypsin, 1 hour before electrophoretic analysis.

\section{IMMUNOCHEMICAL ANALYSIS}

Polyvalent antisera to meconium antigens of nonplasma origin were raised in rabbits as previously described. ${ }^{8}$ Monospecific antisera to CEA were obtained from DAKO-Immunoglobulins, Copenhagen, Denmark. This antiserum detected CEArelated substances and was not specific for CEA.

Monospecific antisera to Mec 1 and Mec 6 were raised in rabbits using partially purified material as follows. Mec 1 and Mec 6 were partially purified by ammonium sulphate precipitation and gel chromatography, ${ }^{8}$ and after immunoelectrophoresis against polyvalent antiserum to meconium antigens and extensive washing of the developed immunoplate, the appropriate precipitation lines were cut out and used as sources of antigen. ${ }^{10}$

Meconium antigens other than alkaline phosphatase were assayed by rocket immunoelectrophoresis using monospecific antisera. ${ }^{9}$ Alkaline phosphatase was measured by immunoelectrophoresis with polyvalent antiserum against meconium antigens and then staining for the 'rockets' containing alkaline phosphatase activity. ${ }^{11} \mathrm{~A}$ meconium standard was prepared from a mixture of specimens collected from 10 healthy infants. Each of the four meconium antigens was given a value of 100 arbitrary units (AU) per gram dry weight in this standard mixture, and the concentrations of the meconium antigens in each test specimen were compared with those in the standard. To date, two of the meconium antigens, Mec 1 and Mec 6, have been isolated; $100 \mathrm{AU} / \mathrm{g}$ dry wt was equivalent to $23 \mathrm{mg} / \mathrm{g}$ dry wt and $29 \mathrm{mg} / \mathrm{g}$ dry wt, respectively. 
STATISTICAL ANALYSIS OF RESULTS

The arithmetic mean and standard deviation of each component in the four groups were calculated, and the results are presented in the appropriate tables. In most cases, however, the distributions were skew. Distributions acceptable for Student's $t$ test analysis were obtained if values were logarithmically transformed before the mean and standard deviation were estimated.

\section{Results}

EFFECT OF PROTEOLYSIS ON ANTIGEN

\section{CONTENT}

There was an insignificant change in the mean alkaline phosphatase and CEA contents after proteolysis of specimens in all four groups. Similarly, the mean Mec 1 contents were only slightly increased after proteolysis of groups 1 and 4 specimens although there was a $30 \%$ increase in group 2 and a $60 \%$ increase in group 3 mean Mec 1 contents. Proteolysis also resulted in a large increase in the mean soluble Mec 6 contents with a twofold increase in groups 1 and 3 and a $40 \%$ increase in group 2 . There was, however, only an insignificant increase in the mean Mec 6 content of group 4 specimens. Analysis by gel chromatography of the four antigens indicated that in no case did proteolysis result in a change of molecular size.

\section{DIFFERENCE IN ANTIGEN CONCENTRATIONS BETWEEN GROUPS AND ITS APPLICATION FOR CF SCREENING}

Proteolysis usually resulted in an increase of the soluble antigen concentration, and consequently a closer representation of the true antigen contents was estimated after proteolysis of meconium. Thus, unless otherwise stated, concentration values were estimated after specimens had been treated with trypsin. Group 1 specimens can be considered as the control group to which changes in the other three groups were compared. All antigen concentrations were significantly lower in group 3 compared to group 1 ( $\mathrm{P}<0.001$ in all cases) (Fig. 2), confirming the original immunoelectrophoretic observation (Fig. 1), but only Mec 6 and CEA mean contents were significantly reduced in the other $C F$ group (group 4). As one of the primary purposes of this study was to determine whether measurements of the four antigens would be of value in screening and reduce the false negative incidence obtained by albumin estimation, further analysis of the results was limited to CEA and Mec 6.

Taking the upper limit of distribution of the two antigens in group 4 as the cut-off point, all CEA values less than $95 \mathrm{AU} / \mathrm{g}$ dry wt and all $\mathrm{Mec} 6$ values estimated after proteolysis of less than $145 \mathrm{AU} / \mathrm{g}$ dry wt would be positive. On these criteria, all specimens in group 3 and $90 \%$ of specimens in group 2 would be positive with respect to both antigens. Of group 1, $17(42 \%)$ specimens would have a positive CEA result and five $(12.5 \%)$ a positive Mec 6 result. Thus the false negative results were eliminated but the false positive incidence was increased.

If, on the other hand, the lowest limit of distribution of the two antigens in group 1 was taken as the cut-off point, all values of CEA below $33 \mathrm{AU} / \mathrm{g}$ dry wt and all values of Mec 6 below 120 AU/g dry wt would be positive. Thus $12(43 \%)$ group 2 specimens, $24(88 \%)$ group 3 specimens, and one $(10 \%)$ group 4 specimen would be positive with regard to CEA content. Similarly, $24(86 \%)$ group 2, $27(100 \%)$ group 3, and seven $(64 \%)$ group 4 specimens had positive Mec 6 values. In this analysis, Mec 6 estimation slightly reduced the false positive incidence and markedly although not totally reduced the false negative incidence.

Apart from routine screening, antigen estimation might be of value in specimens from infants known to be at risk but whose specimen gave a negative result with albumin screening. Seven infants in group 4 were known to be at risk before birth and had Mec 6 values between 44 and 127 AU/g dry wt. Specimens were also examined from nine younger sibs of CF children, subsequently shown by sweat tests to be free of the disease. Five had Mec 6 values less than $145 \mathrm{AU} / \mathrm{g}$ dry wt; two of these four specimens were from preterm twins (see below). The meconium from the two infants considered to have reached term had Mec 6 values of 115 and $130 \mathrm{AU} / \mathrm{g}$ dry wt.

\section{CHANGES OF ANTIGEN CONCENTRATION IN} MECONIUM DURING GESTATION

The reduced level of antigens in group 2 specimens, which were from preterm infants, led to the investigation of the relation of gestational age to the concentration of the antigens. Antigens were measured in specimens collected at various gestational ages (Table).

The Mec 1 concentration reached $75 \%$ of the full-term level by mid-term and more than $90 \%$ soon after, whereas alkaline phosphatase concentration rose slowly throughout gestation, reaching the highest level a month before term. CEA was not detected in meconium until after mid-term, when it was found at $75 \%$ of its final level. Analysis of 2-3-month-old fetal gut tissue showed that CEA was present and thus, although synthesised in the gut at an early gestational age, it was not secreted until much later. Mec 6 concentration rose slowly 

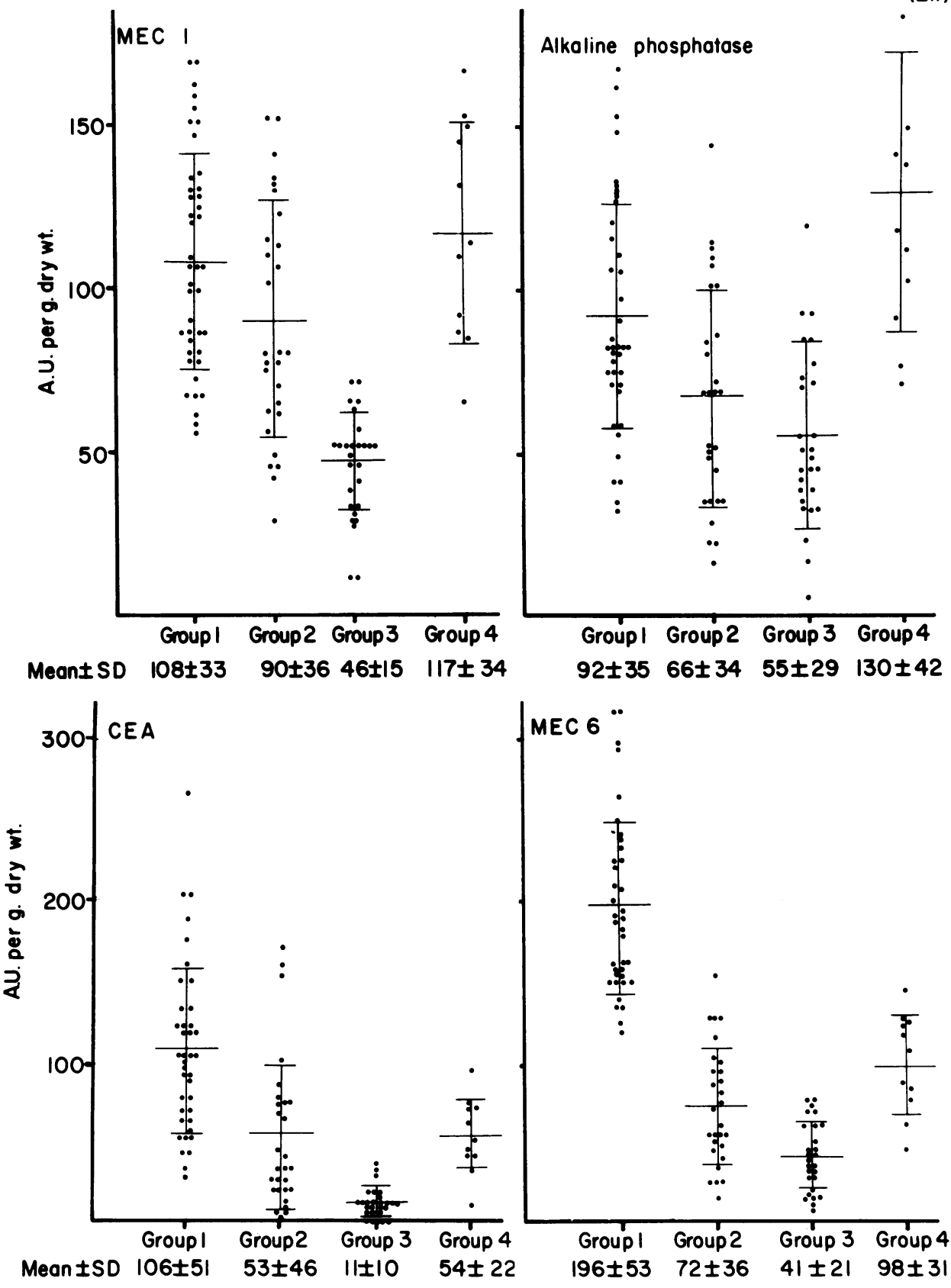

Fig. 2 Distribution of the four meconium antigens in the four groups of specimens: group $1(n=40)$; group $2(n=28) ;$ group $3(n=27) ;$ group $4(n=11)$. Groups 1 and 2 specimens were from healthy infants; groups 3 and 4 specimens were from neonates with $C F$ (see text). 
Distribution of non-plasma antigens in meconium during gestation

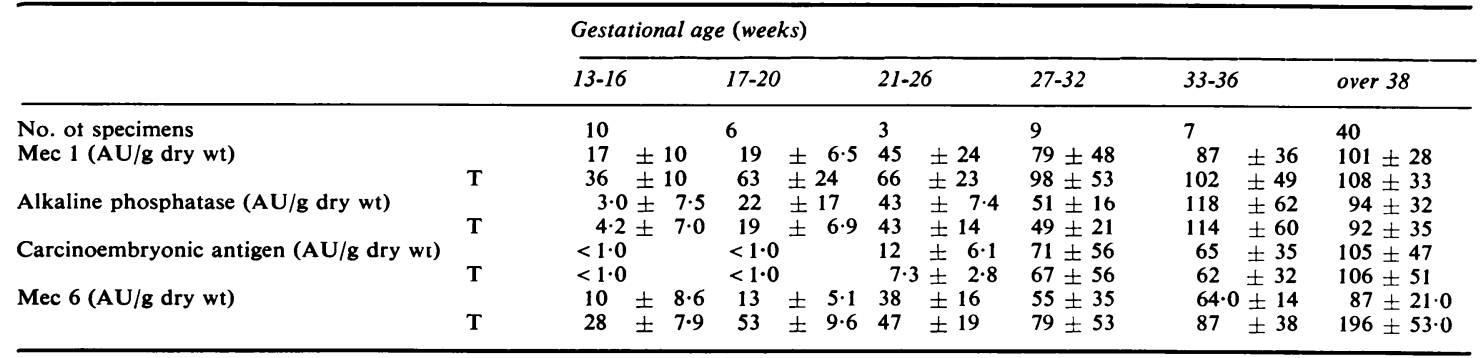

$\mathbf{T}=$ after trypsin digestion of specimen; $\mathbf{A U}=$ arbitrary units.

throughout gestation with a sudden twofold increase in the last month.

FACTORS OTHER THAN UNDERLYING PATHOLOGY THAT MIGHT AFFECT THE ANTIGEN CONCENTRATION IN MECONIUM The differences between group 1 and groups 3 and 4 may reflect the presence of the underlying CF lesion, but there are at least two other possibilities that need to be considered. Firstly, all antigens were estimated on the basis of a dry weight, but proteolytically labile serum protein, absent in group 1 specimens, contributed to the dry weight of both groups 2 and 3 specimens. Thus there was a 'dilution' of the non-meconium antigens by serum protein in groups 2 and 3 specimens. Secondly, the specimens in groups 3 and 4 are from children of various gestational ages, and the changes seen in these groups might, in part, be due to the disparity of gestational age.

Antigen content in groups 2 and 3 specimens corrected for 'serum protein dilution'

The antigen concentrations in groups 2 and 3 were recalculated using a dry weight that had been corrected for the presence of proteolytically labile serum protein content. For this correction, proteolytically labile serum protein was taken as the sum of the albumin and transferrin content in a specimen; these two proteins represent $95-100 \%$ of such protein in meconium. This weight was subtracted from the dry weight, and the antigen content was recalculated per gram corrected dry weight.

The corrected mean Mec 1 concentrations in groups 2 and 3 were $108 \pm 45$ and $71 \pm 23 \mathrm{AU} / \mathrm{g}$ dry wt respectively, and only the mean Mec 1 concentrations in groups 1 and 3 were still significantly different $(P<0.001)$. Alkaline phosphatase concentrations were still not significantly different between the groups. Corrected CEA and Mec 6 concentrations of $65 \pm 54$ and $94 \pm 45 \mathrm{AU} / \mathrm{g}$ dry $w \mathrm{t}$ in group 2 and of $21 \pm 16$ and $68 \pm 36 \mathrm{AU} / \mathrm{g}$ dry $w t$ in group 3 were still significantly lower than in group $1(\mathrm{P}<0.001)$.

\section{Effect of gestational age on composition of CF} meconium

The importance of gestational age was not recognised until a number of $\mathrm{CF}$ specimens had been collected, and a gestational age was not always supplied with specimens sent from outside the Cardiff area. Consequently, of the 27 specimens in group 3, only 10 specimens were known to have come from children born at term and four specimens from preterm infants. The mean concentrations of the antigens in the 10 full-term specimens was: Mec $1,42 \pm 15$; alkaline phosphatase, $56 \pm 44$; CEA, $11 \pm 11$ : and Mec 6, $42 \pm 22 \mathrm{AU} / \mathrm{g}$ dry wt. These concentrations were not significantly different ( $P>0 \cdot 1)$ when compared to either the mean concentration of the whole group or the group minus the 10 specimens. Similarly, in group 4 , six specimens were known to come from full-term infants and two from pre-term infants. The mean concentrations of the antigens in the six specimens were: Mec 1, $114 \pm 41$; alkaline phosphatase, $138 \pm 38$; CEA, $51 \pm 28$ : and Mec 6, $99 \pm 37$ AU/g dry wt. Again no significant difference was found between the six specimens and the whole group or the remaining five specimens. Therefore, in this study, the gestational age of CF specimens appears to be of little importance.

\section{Discussion}

The macromolecular components of meconium can be divided into three groups: serum proteins of maternal origin, mucin, and non-mucin antigens of alimentary tract origin. 5 The distribution of the third group of meconium components is reported here. None of the four antigens studied was present in duodenal aspirates whereas three of the antigens, alkaline phosphatase, $\operatorname{Mec} 1$, and $\operatorname{Mec} 6$, have been detected in the faeces of young children (unpublished work). Therefore these components appear to be of intestinal origin, and changes in their meconium distribution may reflect changes in intestinal secretions. 
Two of the antigens determined, CEA and Mec 6, had mean concentrations in the two CF groups significantly lower than in meconium of group 1 . The mean concentrations in group 2 specimens were also significantly lower than in group 1 , and this meant that their measurement would be of little value in reducing the 'false positive' incidence. Also, although the mean values of both CEA and Mec 6 were significantly different between groups 1 and 4 , there was an overlap of values between the two groups. Consequently, either the incidence of 'false negative' results obtained after albumin was only partially reduced, or there was an increase in false positive results, depending where the value between positive and negative was placed. Thus there appeared to be little advantage in measuring CEA or Mec 6 as an alternative to the measurement of albumin routine screening. The results, however, indicated that the measurement of Mec 6 after specimen proteolysis might be a useful confirmatory test where meconium from a child, known to be at risk, gave a negative albumin test result. The differences in Mec 6 concentrations in healthy and CF children appeared to be due to an increase in the secretion of Mec 6 in the healthy conceptus just before term but this increase did not occur in the CF conceptus. Therefore the test would be carried out only if the child was born at term and could then only confirm a negative result rather than indicate the presence of CF. Such a test when applied to normal siblings of CF children was only partially successful. Four of the nine specimens from the newborn known to be at risk had values less than $145 \mathrm{AU} / \mathrm{g}$ dry wt; two of the specimens were from infants considered to have reached term. It may be that a more accurate estimation of gestational age than was used in this study is needed. Alternatively, it is possible that Mec 6 concentrations will be lower in meconium from CF heterozygotes than from normal homozygotes.

The distribution of antigens in meconium from the two CF groups was similar to that from infants of early gestational ages. Thus the CEA, Mec 6, and Mec 1 concentrations in group 3 are similar to those at mid-term. The group 4 specimens had normal Mec 1 concentrations, above average alkaline phosphatase concentrations, but only half the expected full-term concentrations of CEA and Mec 6, findings similar to those found in meconium from preterm infants of gestational age 32-36 weeks. As the concentration of antigens in CF meconium was independent of the gestational age at birth, one explanation for this apparent correlation is that the underlying CF lesion in some way affects the maturation of intestinal secretions and, possibly, other mucin secretions as well.

I thank Mrs S Rees and Mr K Ashman for technical assistance at various times during this study, and the nursing staff of the University Hospital of Wales Maternity Unit for conscientious cooperation. This work was supported by a Medical Research Council Grant.

\section{References}

${ }^{1}$ Committee for a study for evaluation of testing for cystic fibrosis. J Pediat 1976;88:711-50.

${ }^{2}$ Prosser R, Owen H, Bull F, Parry B, Smerkinich J, Goodwin HA, Dathan J. Screening for cystic fibrosis by examination of meconium. Arch Dis Child 1974;49: 597-601.

${ }^{3}$ Ryley HC, Neale Lynne M, Brogan TD, Bray PT Screening for cystic fibrosis in the newborn by meconium analysis. Arch Dis Child 1979;54:92-7.

4 Wood RE, Boat TF, Doershuk CF. Cystic fibrosis. Am Rev Resp Dis 1976;113:833-78.

${ }^{5}$ Ryley HC. Meconium: its origins, composition and abnormalities. In: Dodge. Topics in paediatric gastroenterology. Tunbridge Wells: Pitman, 1976.

${ }^{6}$ Antonowicz I, Ishida S, Shwachman H. Studies in meconium: disaccharidase activities in meconium from cystic fibrosis patients and controls. Pediatrics 1975;56: 782-7.

'Antonowicz I, Ishida S, Shwachman H. Studies in meconium in cystic fibrosis: the activities of $\alpha-\mathrm{D}$ mannosidase, $\beta$-glucoronidase, $\alpha$-D-fucosidase, acid and alkaline phosphatase. Biol Neonate 1978;84:225-30.

${ }^{8}$ Ryley HC. Immunoelectrophoretic analysis of human meconium antigens of non-plasma origin. J Chromat 1977;143:411-4.

${ }^{2}$ Ryley HC, Neale Lynne M, Brogan TD, Bray PT. Screening for cystic fibrosis by analysis of meconium for albumin and protease inhibitor. Clin Chim Acta 1975; 64:117-25.

${ }^{10}$ Krøll J, Andersen MM. Specific antisera produced by immunization with precipitin lines. J Immunol Methods 1976;16:125-9.

11 Uriel J. Characterization of precipitates in gels. In: Williams and Chase. Methods in immunology and immunochemistry. Vol. 3. New York and London: Academic Press, $1971 ; 313$.

Requests for reprints to: Dr HC Ryley, Department of Medical Microbiology, Welsh National School of Medicine, Cardiff CF4 4XN. 